\title{
Clinical Outcomes of Non-Atrial Fibrillation Bradyarrhythmias Treated With a Ventricular Demand Leadless Pacemaker Compared With an Atrioventricular Synchronous Transvenous Pacemaker - A Propensity Score-Matched Analysis -
}

\author{
Kenichi Sasaki, MD, PhD; Daisuke Togashi, MD; Ikutaro Nakajima, MD, PhD; \\ Taro Suchi, MD; Yui Nakayama, MD, PhD; \\ Tomoo Harada, MD, PhD; Yoshihiro J Akashi, MD, PhD
}

\begin{abstract}
Background: Implanting a ventricular demand leadless pacemaker (VVI-LPM) for patients with non-atrial fibrillation (AF) bradyarrhythmias such as sick sinus syndrome (SSS) or high-grade (i.e., second- or third-degree) atrioventricular (AV) block is not recommended unless they have limited vascular access or a high infection risk; nevertheless, an unexpectedly high number of VVI-LPM implantations have been performed. This study investigated the clinical outcomes of these unusual uses.
\end{abstract}

\begin{abstract}
Methods and Results: This study retrospectively analyzed 193 patients who were newly implanted with a VVI-LPM or an atrioventricular synchronous transvenous pacemaker (DDD-TPM) for non-AF bradyarrhythmias at a high-volume center in Japan from September 2017 to September 2020. Propensity score-matching produced 2 comparable cohorts treated with a VVI-LPM or DDD-TPM $(n=58$ each). Each group had 20 (34\%) patients with SSS and $38(66 \%)$ patients with high-grade AV block. During a median follow up of 733 (interquartile range 395-997) days, there were no significant differences between the VVI-LPM and DDD-TPM groups regarding late device-related adverse events $(0 \%$ vs. $4 \%$, log-rank $P=0.155)$, but the VVI-LPM group had a significantly increased readmission rate for heart failure (HF) $(29 \%$ vs. $2 \%$, log-rank $P=0.001)$ and a tendency to have higher all-cause mortality $(28 \%$ vs. $4 \%$, log-rank $\mathrm{P}=0.059$ ).
\end{abstract}

Conclusions: The implantation of a VVI-LPM for non-AF bradyarrhythmias increased the incidence of HF-related rehospitalization at the mid-term follow up compared to the use of a DDD-TPM.

Key Words: Clinical outcome; Heart failure; Leadless pacemaker; Tricuspid valve

A ventricular demand (VVI) leadless pacemaker (VVI-LPM) was introduced clinically to address lead- and pocket-related complications such as vascular occlusion, lead infection, lead fracture, and pocket infection in transvenous pacemaker (PM) therapy. ${ }^{1}$ A VVI-LPM is a self-contained right ventricular (RV) single-chamber PM for patients requiring VVI pacing, such as those with atrial fibrillation (AF) with a slow ventricular response (AF-SVR). Originally, for patients with limited vascular access and a high infection risk, the implantation of a VVI-LPM was considered a potential alternative to a transvenous PM; however, an unexpectedly high number of VVI-LPM implantations have been performed, and a VVI-LPM has been used more often in specific cohorts rather than a transvenous PM (e.g., in patients undergoing hemodialysis [HD] and in patients after a transcatheter aortic valve implantation [TAVI]), achieving comparable results.,3

In general, an atrioventricular (AV) synchronous (DDD) transvenous PM (DDD-TPM) is preferred for non-AF bradyarrhythmias such as sick sinus syndrome (SSS) and high-grade (i.e., second- or third-degree) AV block (AVB), because compared to a VVI-LPM, the use of a DDD-TPM can preserve AV synchrony and prevent PM syndrome. Although the inferiority of VVI pacing compared to DDD pacing for patients with non-AF bradyarrhythmia has been established in the use of TPM, ${ }^{4}$ no data are available regarding the VVI pacing provided by a VVI-LPM vs. the

Received October 19, 2021; revised manuscript received December 19, 2021; accepted December 29, 2021; J-STAGE Advance Publication released online January 29, 2022 Time for primary review: 20 days

Division of Cardiology, Department of Internal Medicine, St. Marianna University School of Medicine, Kawasaki, Japan

Mailing address: Kenichi Sasaki, MD, PhD, Division of Cardiology, Department of Internal Medicine, St. Marianna University School of Medicine, 2-16-1 Sugao, Kawasaki 216-8551, Japan. E-mail: kenichi.sasaki@marianna-u.ac.jp

All rights are reserved to the Japanese Circulation Society. For permissions, please e-mail: cj@j-circ.or.jp

ISSN-1346-9843 


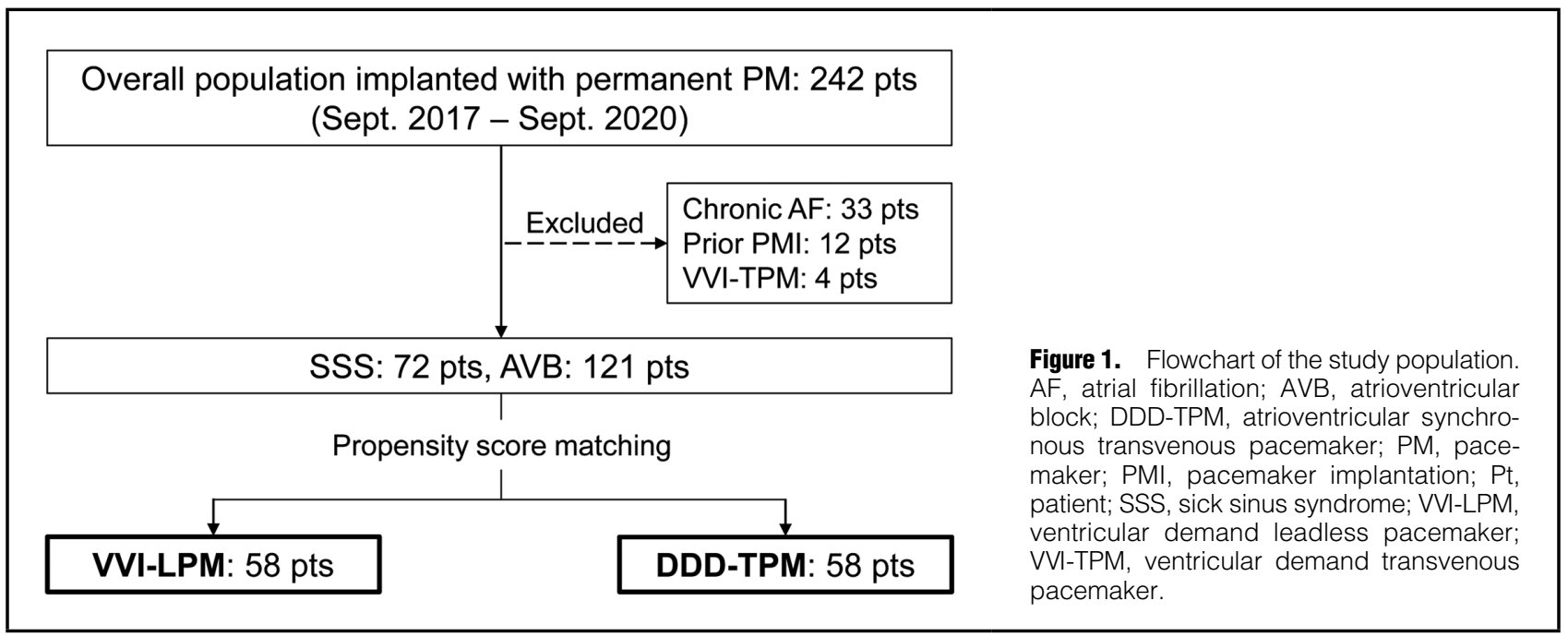

DDD pacing provided by a DDD-TPM. We therefore evaluated the clinical courses of patients in whom a VVI-LPM had been implanted for non-AF bradyarrhythmia, and we compared their courses with those of patients who underwent DDD-TPM implantation. When analyzing the data, we included SSS and AVB in the same group, because the purpose of this study was to focus on the clinical effect of AV asynchronous pacing caused by a VVI-LPM. Moreover, Japanese guideline refers to SSS and AVB as non-AF bradyarrhythmia and provides the dichotomy between AF-SVR and non-AF bradyarrhythmias for VVI-LPM indication. ${ }^{5}$

In our present investigation, the selection of the PM type for patients with non-AF bradyarrhythmia was not randomly assigned; rather, the PM type was chosen according to the treating physician or heart team's decision. We therefore conducted a propensity score (PS)-matched analysis in order to adjust for confounding factors and reduce differences in the clinical characteristics when comparing the VVI-LPM and DDD-TPM groups.

\section{Methods}

\section{Study Design and Population}

This was a single-center, retrospective observational study. We enrolled 242 consecutive Japanese patients who underwent a new permanent PM implantation (PMI) at St. Marianna University Hospital (Kawasaki, Japan) for the treatment of bradyarrhythmias based on Class I or II guideline recommendations ${ }^{6}$ during the period from September 2017 to September 2020. Of these, 33 patients who underwent a PMI for AF-SVR (including 2 patients who changed pacing mode from DDD to VVI because of AF becoming chronic) and 4 patients with a VVI-TPM were excluded. We also excluded 12 patients who experienced reimplantation because of lead failures, device upgrades, or device infection because residual leads would influence the clinical course.

Upgrades were defined as those from a single-chamber pacing device to a dual-chamber pacing device, implantable cardioverter-defibrillator, or cardiac resynchronization therapy device, and cases were excluded even if the complete removal of the prior device system was achieved. A final total of 193 patients were recruited in this study. Using the methods described below, we then calculated the PSs for the PM types and created 58 patient pairs (Figure 1). The data regarding the patients' baseline characteristics, electrocardiogram (ECG) and transthoracic echocardiography (TTE) findings, the PMs' electrical performance, procedural characteristics, and indications for PMI were recorded. The TTE and PM parameters were also collected at each follow up. Significant valvular disease was defined as any severe valvular stenosis or regurgitation. The study conformed with the ethical guidelines of the Declaration of Helsinki, and the protocol was approved by the Institutional Ethics Committee (reference no. 5431).

\section{Leadless and Transvenous PM System}

Standard implantation techniques were used for the DDDTPM. The pacing system of a DDD-TPM consists of a PM device containing the electronics and battery typically implanted in a subcutaneous pocket in the chest region, and 2 leads are advanced from the device pocket through the patient's veins into the right atrium (RA) and the RV.

The only leadless PM available in Japan is the Micra ${ }^{\mathrm{TM}}$ (Medtronic, Minneapolis, MN, USA). Each Micra ${ }^{\mathrm{TM}}$ implantation was performed according to the manufacturer's protocol. ${ }^{7,8}$ The target implant site was the RV mid-septal region. When this region was found to have inadequate pacing and/ or sensing threshold or technical difficulties for deployment, a site in the apical or high RV septum was chosen.

\section{Analysis of ECG and TTE}

After the implantation of a VVI-LPM, 12-lead ECG during ventricular pacing was evaluated for the maximum duration of the QRS. TTE examinations were performed before the implantation and at the scheduled follow ups. The following data were extracted: left ventricular (LV) ejection fraction (LVEF), LV dimension (LVD), the severity of tricuspid valve regurgitation (TR) and mitral valve regurgitation (MR), and the estimated RV systolic pressure (RVSP). The LVEF was calculated using the biplane disc summation method. The diameter of the LV was measured at the level of the mitral valve leaflet tips from the parasternal long-axis image. The grading of TR or MR severity was performed according to the American Society of Echocardiography guidelines ${ }^{9}$ and categorized into five groups: 


\begin{tabular}{|c|c|c|c|c|c|c|}
\hline & \multicolumn{2}{|c|}{$\begin{array}{c}\text { Pre-propensity score } \\
\text { matching }\end{array}$} & \multirow{2}{*}{$P$ value } & \multicolumn{2}{|c|}{$\begin{array}{l}\text { Post-propensity score } \\
\text { matching }\end{array}$} & \multirow{2}{*}{$P$ value } \\
\hline & $\begin{array}{l}\text { VVI-LPM } \\
(n=110)\end{array}$ & $\begin{array}{c}\text { DDD-TPM } \\
(\mathrm{n}=83)\end{array}$ & & $\begin{array}{l}\text { VVI-LPM } \\
(n=58)\end{array}$ & $\begin{array}{l}\text { DDD-TPM } \\
(n=58)\end{array}$ & \\
\hline Age, years & $82 \pm 9$ & $80 \pm 8$ & 0.157 & $81 \pm 8$ & $82 \pm 6$ & 0.791 \\
\hline Female & $65(59)$ & $50(60)$ & 0.883 & $36(62)$ & $35(60)$ & 1.000 \\
\hline Body mass index, $\mathrm{kg} / \mathrm{m}^{2}$ & $22 \pm 3$ & $22 \pm 4$ & 0.341 & $23 \pm 4$ & $23 \pm 3$ & 0.774 \\
\hline Hypertension & $66(60)$ & $48(59)$ & 0.770 & $36(62)$ & $34(59)$ & 0.850 \\
\hline Diabetes mellitus & $17(15)$ & $23(28)$ & 0.048 & $10(17)$ & $16(28)$ & 0.265 \\
\hline Dyslipidemia & $38(35)$ & $33(40)$ & 0.547 & $21(36)$ & $26(45)$ & 0.450 \\
\hline COPD & $3(1)$ & $1(1)$ & 0.636 & $3(5)$ & $1(2)$ & 0.618 \\
\hline eGFR, $\mathrm{mL} / \mathrm{min} / 1.73 \mathrm{~m}^{2}$ & $65 \pm 29$ & $52 \pm 19$ & $<0.001$ & $55 \pm 26$ & $54 \pm 17$ & 0.904 \\
\hline (log-transformed) NT-proBNP, pg/mL & $7.0 \pm 1.4$ & $6.6 \pm 1.7$ & 0.137 & $7.1 \pm 1.5$ & $6.7 \pm 1.6$ & 0.213 \\
\hline \multicolumn{7}{|l|}{ Cardiovascular disease } \\
\hline History of heart failure & $4(4)$ & $4(5)$ & 0.727 & $3(5)$ & $3(5)$ & 1.000 \\
\hline Cardiomyopathy & $0(0)$ & $5(6)$ & 0.014 & $0(0)$ & $3(5)$ & 0.244 \\
\hline Coronary artery disease & $15(14)$ & $17(20)$ & 0.243 & $12(21)$ & $13(22)$ & 1.000 \\
\hline Severe valvular heart disease & $31(28)$ & $31(37)$ & 0.213 & $19(33)$ & $23(40)$ & 0.563 \\
\hline Prior TAVI & $20(18)$ & $23(28)$ & 0.121 & $12(21)$ & $16(28)$ & 0.516 \\
\hline Total & $48(44)$ & $49(59)$ & 0.042 & $33(57)$ & $36(62)$ & 0.706 \\
\hline \multicolumn{7}{|l|}{ Pacing indication } \\
\hline Sick sinus syndrome & $48(44)$ & $24(29)$ & 0.050 & $20(34)$ & $18(31)$ & 0.843 \\
\hline Atrioventricular block & $62(56)$ & $59(71)$ & 0.050 & $38(66)$ & $40(69)$ & 0.843 \\
\hline \multicolumn{7}{|l|}{ 12-lead ECG } \\
\hline Paced QRS duration, ms & $155 \pm 14$ & $161 \pm 14$ & 0.018 & $158 \pm 15$ & $161 \pm 16$ & 0.303 \\
\hline \multicolumn{7}{|l|}{ TTE findings } \\
\hline LV ejection fraction, $\%$ & $64 \pm 9$ & $64 \pm 9$ & 0.801 & $63 \pm 10$ & $63 \pm 9$ & 0.962 \\
\hline LV diastolic diameter, mm & $43 \pm 6$ & $45 \pm 6$ & 0.015 & $43 \pm 6$ & $45 \pm 7$ & 0.126 \\
\hline $\mathrm{TR} \geq$ moderate & $9(10)$ & $8(10)$ & 1.000 & $5(9)$ & $7(12)$ & 0.763 \\
\hline Estimated RV systolic pressure, $\mathrm{mmHg}$ & $30 \pm 9$ & $29 \pm 10$ & 0.543 & $29 \pm 9$ & $30 \pm 11$ & 0.749 \\
\hline
\end{tabular}

Categorical variables are presented as absolute number and percentages of each subgroup. Continuous variables are displayed as mean \pm standard deviation. COPD, chronic obstructive pulmonary disease; DDD-TPM, atrioventricular synchronous transvenous pacemaker; ECG, electrocardiogram; eGFR, estimated glomerular filtration rate; LV, left ventricle; NT-proBNP, N-terminal pro-B-type natriuretic peptide; $\mathrm{RV}$, right ventricle; TAVI, transcatheter aortic valve implantation; TR, tricuspid valve regurgitation; TTE, transthoracic echocardiography; VVI-LPM, ventricular demand leadless pacemaker.

$0=$ none or trivial, $1=$ mild, $2=$ mild to moderate, $3=$ moder ate to severe, and $4=$ severe. The degree of TR was based on the color flow jet area in the RA observed by using the apical 4-chamber view in addition to the continuous-wave Doppler pattern, the vena contracta width, and the hepatic vein flow. Continuous-wave Doppler of the TR jet was used for the estimation of the RVSP using the modified Bernoulli equation and RA pressure, which was estimated in consonance with the inferior vena cava size. For the determination of the degree of MR, quantitative data from color Doppler involving the color flow jet area in the left atrium and pulmonary vein flow were used.

\section{Follow up and Study Endpoint}

The patients were retrospectively followed up until September 2021. Follow ups at the outpatient clinic were scheduled at 1,6 , and 12 months post-PMI and annually thereafter. Clinical information of patients who did not undergo regular checkups was obtained by telephone interviews or contact with the patient's primary care physician. The RV and atrial pacing burden that we used for analysis was the percentage at the 12-month follow up (or the last device check-up if the duration after PMI was within 1 year).

Because setting the lower ventricular pacing rate at a higher level led to a higher ventricular pacing percentage $\left(\mathrm{VP}^{0} \%\right)$, we adjusted the true $\mathrm{VP} \%$ by using the following formula: the true $\mathrm{VP} \%$ divided by (the set lower rate divided by 60 beats/min, which was the general lower rate), and we thereby calculated the corrected $\mathrm{VP} \%(\mathrm{cVP} \%)$. The maximum value of the $\mathrm{cVP} \%$ was defined as $100 \%$. Similar processing was performed for atrial pacing burden.

We determined the study endpoints as follows: (1) allcause mortality; (2) cardiovascular mortality; (3) rehospitalization for heart failure (HF); and (4) late device-related adverse events (AEs) during the follow-up period. Any death of unknown cause was considered a cardiovascular mortality. Rehospitalization for HF was defined as any new unplanned overnight stay in a hospital because of worsening signs and/or symptoms of HF that required an intensification of medical or mechanical therapy after PMI. The conclusive diagnosis of HF was made by attending physicians. Late device-related AEs were defined as all accidents (e.g., device infection, elevated threshold, lead dislodgement, lead failure, or pericardial effusion) that were attributed to the device or implantation procedure and occurred after the patient's discharge. Elevation of the pacing threshold was defined as an increase by more than 2-fold from the value when the PM was implanted. 


\begin{tabular}{|c|c|c|c|c|c|c|}
\hline & \multicolumn{2}{|c|}{$\begin{array}{l}\text { Pre-propensity score } \\
\text { matching }\end{array}$} & \multirow{2}{*}{$P$ value } & \multicolumn{2}{|c|}{$\begin{array}{l}\text { Post-propensity score } \\
\text { matching }\end{array}$} & \multirow{2}{*}{$P$ value } \\
\hline & $\begin{array}{l}\text { VVI-LPM } \\
(n=110)\end{array}$ & $\begin{array}{c}\text { DDD-TPM } \\
(\mathrm{n}=83)\end{array}$ & & $\begin{array}{c}\text { VVI-LPM } \\
(n=58)\end{array}$ & $\begin{array}{c}\text { DDD-TPM } \\
(\mathrm{n}=58)\end{array}$ & \\
\hline \multicolumn{7}{|l|}{ Ventricular pacing site } \\
\hline RV septum & $109(99)$ & 0 & & $58(100)$ & 0 & \\
\hline RV apex & $1(1)$ & $82(99)$ & & 0 & $57(98)$ & \\
\hline RV outflow & 0 & $1(1)$ & & 0 & $1(2)$ & \\
\hline Fluoroscopic time, min & $7(5-9)$ & $8(5-15)$ & 0.161 & $5(7-10)$ & $5(9-17)$ & 0.323 \\
\hline Admission days after PMI, days & $4(2-7)$ & $5(4-8)$ & $<0.001$ & $4(2-7)$ & $5(4-8)$ & 0.002 \\
\hline \multicolumn{7}{|l|}{ Adverse events in hospital } \\
\hline Cardiac tamponade & 0 & 0 & & 0 & 0 & \\
\hline Hematoma & 0 & 3 & & 0 & 3 & \\
\hline Pneumothorax/hemothorax & 0 & 0 & & 0 & 0 & \\
\hline Arterial injury/arteriovenous fistula & 2 & 0 & & 2 & 0 & \\
\hline Infection & 0 & 0 & & 0 & 0 & \\
\hline Lead or pacemaker dislocation & 0 & 2 & & 0 & 2 & \\
\hline Elevated pacing threshold & 1 & 2 & & 0 & 2 & \\
\hline Total & $3(3)$ & $7(8)$ & 0.103 & $2(3)$ & $7(12)$ & 0.162 \\
\hline VP\% at 12 -month or the last PM check & $47(4-91)$ & $74(2-99)$ & 0.571 & $51(5-93)$ & $70(1-99)$ & 0.900 \\
\hline cVP\% at 12 -month or the last PM check & $45(6-83)$ & $75(3-99)$ & 0.076 & $51(6-82)$ & $75(2-99)$ & 0.216 \\
\hline $\mathrm{AP} \%$ at 12 -month or the last PM check & & $38(10-79)$ & & & $38(10-72)$ & \\
\hline cAP\% at 12 -month or the last PM check & & $38(10-79)$ & & & $38(10-72)$ & \\
\hline
\end{tabular}

Categorical variables are presented as absolute number and percentages of subgroup. Continuous variables are displayed as median (Q1Q3). AP\%, atrial pacing percentage; cAP\%, corrected atrial pacing percentage; cVP\%, corrected ventricular pacing percentage; DDD-TPM, atrioventricular synchronous transvenous pacemaker; PMI, pacemaker implantation; RV, right ventricle; VP\%, ventricular pacing percentage; VVI-LPM, ventricular demand leadless pacemaker.

\section{Statistical Analysis}

Continuous variables are presented as the mean \pm standard deviation or the median (interquartile range [IQR]) and were compared using Student's t-test or the Wilcoxon rank-sum test depending on the variable's distribution. Categorical values are presented as counts and percentages and were compared using Fisher's exact test. The cumulative incidences of clinical events at follow up were assessed using the Kaplan-Meier method and log-rank test. The PS for device type (i.e., VVI-LPM or DDD-TPM) was obtained from a logistic regression model using the following 6 covariates: age, sex, estimated glomerular filtration rate (eGFR), LVEF, RVSP, and PM indication (i.e., SSS or AVB). The patients in the VVI-LPM and DDD-TPM groups were matched on a 1:1 basis with a 4-digit nearest neighbor algorithm within a caliper of 0.2 . Two-sided $\mathrm{P}$ values $<0.05$ were considered significant. Statistical analyses were performed using $\mathrm{JMP}^{\circledR} 14$ (SAS Institute Inc., Cary, NC, USA).

\section{Results}

\section{Patient Characteristics Before and After PS Matching}

The baseline characteristics of the VVI-LPM and DDDTPM groups before and after PS matching are summarized in Table 1. Before matching, the VVI-LPM group had significantly higher eGFR values and were more significantly likely to have diabetes mellitus and heart disease. Regarding the pacing indication, there was a tendency for a higher proportion of SSS cases in the VVI-LPM group (44\%) compared to the DDD-TPM group $(29 \%)(\mathrm{P}=0.050)$. With regard to 12-lead ECG before PS matching, the paced QRS durations in the VVI-LPM group were significantly narrower than those in the DDD-TPM group $(155 \pm 14 \mathrm{~ms}$ vs. $161 \pm 14 \mathrm{~ms}, \mathrm{P}=0.018$ ). This is because the VVI-LPMs were deployed in the septal site when possible, whereas all but one of the DDD-TPMs in this patient series were implanted at the apex. Regarding the TTE parameters, significantly larger LV diastolic diameters were observed in the DDDTPM group before PS matching $(45 \pm 6 \mathrm{~mm}$ vs. $43 \pm 6 \mathrm{~mm}$, $\mathrm{P}=0.015$ ); however, no significant between-group differences for any covariates were observed after PS matching.

\section{Indications for the VVI-LPM}

The selection of the type of PM (i.e., VVI-LPM or DDDTPM) to be implanted was determined by the patient's attending physician or an arrhythmia heart team. Generally, a VVI-LPM was implanted if there were unfavorable conditions for a DDD-TPM: (1) susceptibility to device infection due to the patient's frailty, steroid use, or other pathology $(n=19)$; (2) preserving venous access for HD $(n=6)(3)$; concern for patients with dementia or delirium who may interfere with the PM pocket, $(n=3)$; (4) a cosmetic issue $(n=2)$; and (5) short life expectancy $(n=1)$. In 6 patients, there was no specific reason for avoiding the selection of a DDD-TPM. In the remaining 21 patients, the indications were based on the physician's prediction that the treated arrhythmia would not need constant ventricular pacing, such as bradycardia-tachycardia syndrome or intermittent AVB.

\section{Device-Related Parameters}

In-hospital, device-related AEs occurred in $8 \%(n=9)$ of the matched cohorts (Table 2). Of these, several complica- 


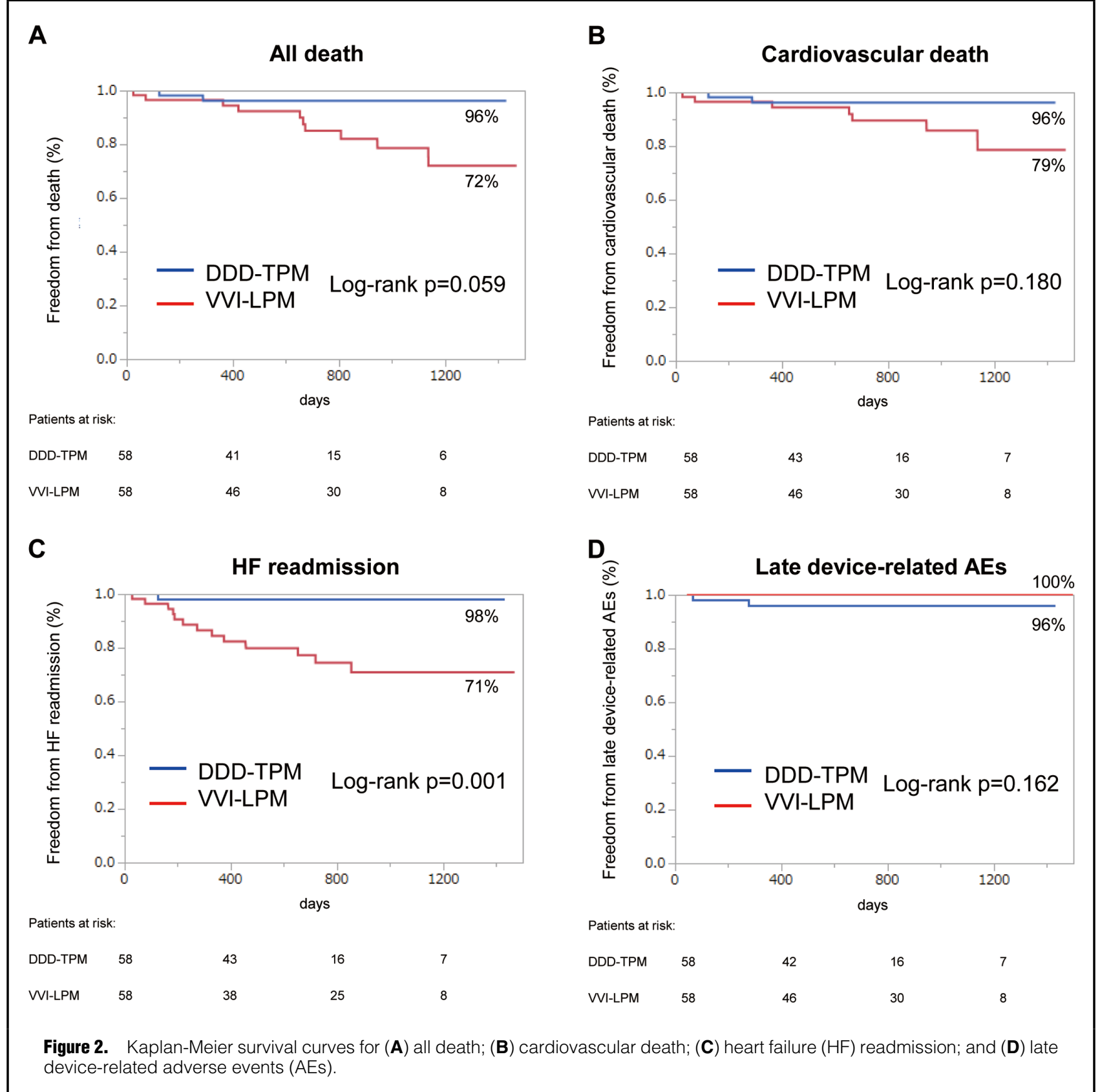

tions specific to each PM type were observed: 3 cases of PM-pocket hematoma and 2 cases of lead dislodgement in the DDD-TPM group, and 1 case of arteriovenous fistula and 1 case of pseudo-aneurysm in the VVI-LPM group.

The VVI-LPM group had a significantly lower number of admission days than the DDD-TPM group: 4 (IQR 2-7) days vs. 5 (IQR 4-8) days, $\mathrm{P}=0.002$. This was because a longer observation for the PM pocket and lead dislocation were not needed in the implantation of the VVI-LPMs compared to the DDD-TPMs.

There were no significant differences between the VVILPM and DDD-TPM groups for the VP\% (51\% [IQR 5-93\%] vs. $70 \%$ [IQR 1-99\%], $\mathrm{P}=0.900)$ or $\mathrm{cVP} \%(51 \%$ [IQR 6-82\%] vs. 75\% [IQR 2-99\%], $\mathrm{P}=0.216)$ values at the 12-month or the last check-up visit. We suspect that these extreme variations in the pacing percentage might represent the difference in VP\% between SSS and AVB; that is, lower $\mathrm{VP} \%$ values in SSS and higher $\mathrm{VP} \%$ values in AVB.

\section{Overall and Cardiovascular Mortality}

The median follow-up durations were 801 days (IQR 4471,124 days) in the VVI-LPM group and 649 days (IQR 375-808 days) in the DDD-TPM group ( $\mathrm{P}=0.069)$. A total of 12 patients had died; non-cardiovascular death $(n=3)$ and cardiovascular death $(n=9)$. The causes of the cardiovascular deaths included sudden death $(n=8)$ and decompensated HF $(n=1)$. During the 48-month follow-up period, there was a non-significant tendency of higher overall mortality $(28 \%$ vs. $4 \%, \log$-rank $\mathrm{P}=0.059)$ in the VVI-LPM group (Figure 2A). 


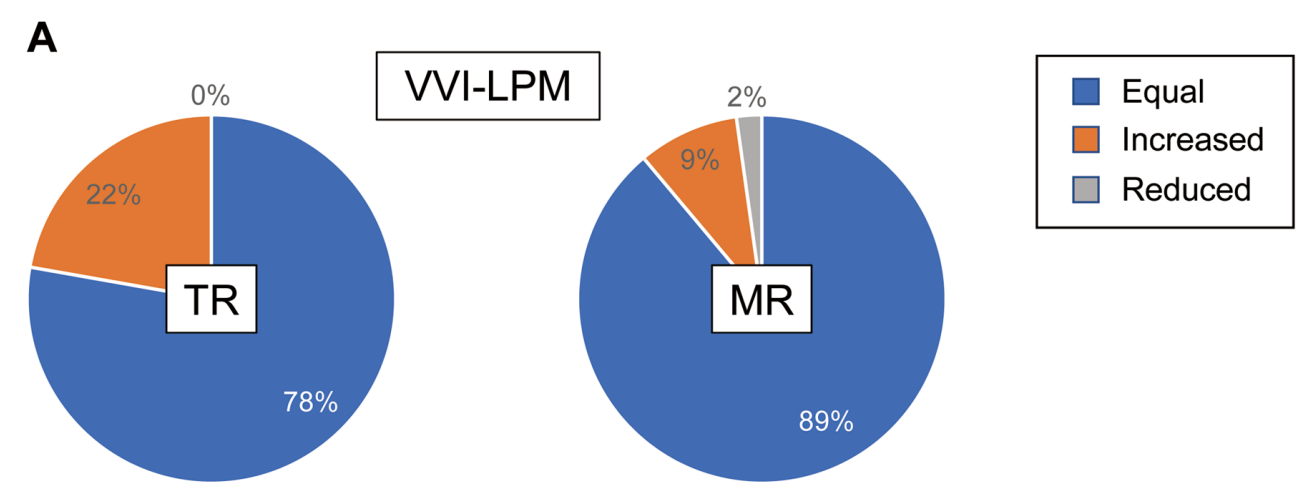

B

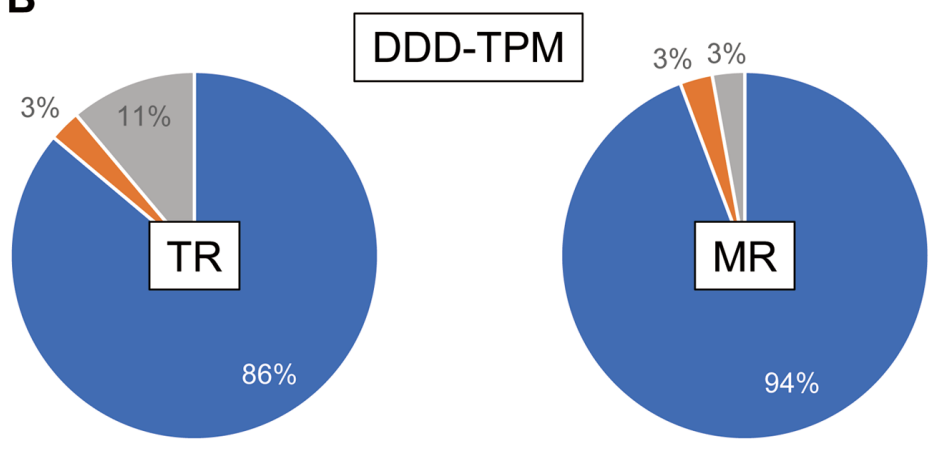

Figure 3. Development of tricuspid valve regurgitation (TR) and mitral valve regurgitation (MR) in the patients with (A) a ventricular demand leadless pacemaker (VVI-LPM) or (B) an atrioventricular synchronous transvenous pacemaker (DDD-TPM). If the severity of regurgitation worsened or improved by $\geq 2$ grades, the severity was defined as 'increased' or 'decreased', respectively. Within a 1 grade change, the severity was defined as 'equal'.

\section{Rehospitalization for HF}

Over 48 months, the Kaplan-Meier analysis showed a significantly higher readmission rate for HF in the VVI-LPM group compared to the DDD-TPM group ( $29 \%$ vs. $2 \%$, $\log$-rank $\mathrm{P}=0.001$; Figure 2C). The median time for repeat hospitalization was 247 days (range 28-854 days). The triggers for decompensated $\mathrm{HF}$ were ventricular pacing-related cardiac malfunction $(n=4)$, severe or worsening valvular heart disease $(n=3)$, dehydration failure in $\operatorname{HD}(n=2)$, overdosage of $\beta$-blockers $(n=1)$, rapid $\mathrm{AF}(\mathrm{n}=1)$, paravalvular leak after aortic valve replacement $(n=1)$, and an unknown cause $(n=2)$. Pacing-related malfunctions included loss of AV synchronous contraction, and pacing-induced cardiomyopathy, which was defined as a $>10 \%$ drop in LVEF in the setting of chronic RV pacing dependency. Of the 4 cases with these morbid states, we upgraded 2 cases to receive cardiac resynchronization therapy and 2 cases to receive a DDD-TPM.

\section{Device-Related Adverse Events After Discharge}

At 48 months, there was no significant difference between the VVI-LPM and DDD-TPM groups for late devicerelated AEs ( $0 \%$ vs. $4 \%$, log-rank $P=0.162$; Figure 2D). All AEs in the DDD-TPM group were associated with device infection, and the lengths of time from the implantation to the events were 66 days and 277 days, respectively.

\section{Development of TR and MR}

Figure 3 shows the development of TR and MR in the
VVI-LPM and DDD-TPM groups. If the severity of regurgitation worsened or improved by $\geq 2$ grades between the value observed at baseline and the value obtained at the 12-month or last follow up, the severity was defined as 'increased' or 'decreased', respectively. A change in severity within 1 grade was defined as 'equal'. A significantly greater proportion of the VVI-LPM patients developed increased TR compared to the DDD-TPM group after PMI $(\mathrm{P}=0.005)$, whereas the MR development in the 2 groups was similar $(\mathrm{P}=0.538)$.

\section{Discussion}

\section{Main Findings}

To the best of our knowledge, this is the first report on clinical outcomes of patients with non-AF bradyarrhythmia who received a VVI-LPM. Our study's PS-matched comparison between the VVI-LPM and DDD-TPM groups revealed that: (1) the implantation of a VVI-LPM for nonAF bradyarrhythmias was associated with a significantly higher incidence of rehospitalization for HF, although it did not increase the risk of overall or cardiovascular mortality compared to the use of a DDD-TPM; (2) the device- and procedure-related complication rates during the follow-up period were both low, especially in the VVI-LPM group; and (3) according to the TTE findings, the aggravation of TR after PMI occurred more frequently in the patients with a VVI-LPM than those with a DDD-TPM. 


\section{A Tendency for Increased Mortality in the VVI-LPM-Implanted Patients}

There was no significant difference between the VVI-LPM and DDD-TPM groups in terms of all-cause mortality or cardiovascular mortality. Previous reports described the safety or electrical performance of a VVI-LPM as comparable to that of a DDD-TPM in the short-term follow up, ${ }^{10-14}$ but little is known about the clinical outcomes of VVI-LPMs in the mid- to long-term, or about the clinical outcomes of VVI-LPM use for non-AF bradyarrhythmia. ${ }^{15}$

In our examination of the Kaplan-Meier survival curves obtained herein, we observed that the rates of overall and cardiovascular death appeared to be similar in the VVILPM and DDD-TPM groups early in the follow up, but then the survival curves tended to dissociate from each other and the mortality rate increased in the VVI-LPM group. Sudden cardiac death (SCD) or death due to an unknown cause accounted for approximately two-thirds of all deaths in our patient population (6 cases in the VVILPM group and 2 in the DDD-TPM group). If there was a relationship between the VVI pacing provided by a VVILPM and SCD, it might result from a reduction of the stroke volume due to AV dyssynchrony, the development of QT dispersion due to ventricular pacing, or a loss of ventricular capture due to an elevated threshold; however, these theories remain a matter of speculation.

VVI pacing for SSS or AVB was reported to be associated with a higher incidence of AF or low health-related quality of life or with poor exercise capacity compared to DDD pacing, but no superiority of DDD pacing was observed regarding mortality or the prevention of stroke or HF, despite its superiority in hemodynamics. ${ }^{16,17}$ Additional observations and populations are needed to clarify the tendency for higher mortality in the VVI-LPM group observed in our study.

\section{Factors Associated With HF Rehospitalization}

The incidence of HF readmission was significantly higher in the VVI-LPM group. Although the episodes of decompensated HF had various triggers, several essential factors associated with this finding should be considered. First, as mentioned above, VVI pacing lacks AV synchrony; as a result, the stroke volume decreases, the systolic blood pressure tends to be low, and the arterial blood lactate value rises during VVI pacing, especially during exercise. ${ }^{18,19}$

Second, the absence of AV synchrony could cause diastolic MR and increase the left atrial pressure, resulting in congestion. Diastolic MR occurs when atrial contraction is not followed by an appropriately timed ventricular systole. ${ }^{20}$ Although we did not perform an assessment of diastolic MR in this study, this unfavorable phenomenon might contribute significantly to the development of $\mathrm{HF}$, considering the hemodynamics provided by VVI pacing.

Third, it is possible that the presence of a VVI-LPM worsens valvular and biventricular function. Beurskens et al reported that the use of a VVI-LPM seemed to adversely influence mitral valve and biventricular function due to $\mathrm{RV}$ pacing-induced ventricular dyssynchrony. ${ }^{21}$ In fact, a substantial reduction ( $>10 \%$ reduction) in LVEF was usually observed in our study population. The VVI-LPM and DDD-TPM groups had similar proportions of patients with reduced LVEF ( $20 \%$ vs. $17 \%, \mathrm{P}=0.779)$. It should be noted that we observed extremely reduced LVEF in 2 of the VVI-LPM patients (from $61 \%$ to $14 \%$ and $54 \%$ to $29 \%$, respectively) with a follow up of $\sim 12$ months. Although it remains unclear why LV dysfunction acutely developed, we speculate that the RV pacing was probably responsible, because the patients with LVEF reduction had significantly higher $\mathrm{cVP} \%$ compared to those without it $(86 \%$ [IQR 40-99\%] vs. 34\% [IQR 3-81\%], $\mathrm{P}=0.029)$. The data regarding clinical outcomes dependent on ventricular pacing burden in the cohort before PS matching are depicted in Supplementary Figure. Finally, new-onset AF or the worsening of an AF attack could have been introduced by VVI pacing and thus cause congestive HF. Although the superiority of DDD pacing compared to VVI pacing in terms of new-onset AF has been described, ${ }^{22,23}$ an $\mathrm{AF}$ attack was seldom responsible for HF readmission in our study population ( $7 \%$ of the total cases).

\section{Worsening TR After VVI-LPM Implantation}

There are conflicting descriptions of worsening TR after VVI-LPM implantation. ${ }^{21,24}$ In our present patient series, the aggravation of TR occurred in $22 \%$ of the VVI-LPM group, which is a lower prevalence compared to the $43 \%$ that Beurskens et al ${ }^{21}$ reported. The reason for our study's lower prevalence is that we defined aggravation as worsening by at least 2 grades, whereas Beurskens et $\mathrm{al}^{21}$ defined it as a change by 1 grade. The differences among previous reports and ours might be due to such differences in grading, the timing of assessment, and/or interobserver variability.

There are several potential reasons why TR more frequently worsened in the present study patients implanted with a VVI-LPM compared to those implanted with a DDD-PM. First, during its implantation, a VVI-LPM could induce tricuspid valve (TV) damage such as leaflet laceration, chordal tearing, and papillary muscle injury. However, an LPM-induced TV injury is rare, and the procedures in the present study patients were performed by experienced operators. Worsening TR due to implant-related complications is thus unlikely.

Second, an LPM could mechanically interfere with the TV or its subvalvular apparatus, which was considered to be the primary cause of worsening TR by Beurskens et al. ${ }^{21}$ It seems likely that the device near the TV may directly compress the leaflets or entangle the chordae tendineae and prevent adequate leaflet coaptation. Unfortunately, we were unable to investigate these potential mechanisms in the present study because the precise observation of the TV or subvalvular apparatus is difficult with conventional 2-dimensional echocardiography; it is necessary to use 3-dimensional echocardiography or other high-resolution modalities to evaluate TR after the implantation of a leadless PM. ${ }^{25,26}$

Third, RV pacing itself may be responsible for its detrimental effect on TR. Delayed activation of the papillary muscles caused by an abnormal RV contraction not via the intrinsic conduction system could induce TR. However, we did not observe a significant relationship between the degree of RV pacing $(\mathrm{cVP} \%)$ and worsening TR in the prePS matching cohort; 78\% (IQR 31-86\%) in the patients with worsening TR vs. 40\% (IQR 3-86\%) in those without $(\mathrm{P}=0.189)$. Finally, we should consider the deleterious effect of AV asynchronous pacing on TR. Data regarding the aggravation of TR during VVI pacing are very scarce. ${ }^{27}$ An investigation into whether RV pacing caused by VVILPM is related to TR should compare the TR during VVI-LPM pacing with that during AV synchronous LPM pacing; such an investigation is our next goal. 


\section{Proper Use of a VVI-LPM as an Alternative to a DDD-TPM}

According to the latest guidelines for arrhythmia in Japan, ${ }^{5}$ a VVI-LPM is recommended as a class-1 indication for patients with AF-SVR and limited vascular access. In other cases, a VVI-LPM will be considered when the patient is frail, very elderly, susceptible to infection due to skin trouble, or has a short life expectancy. We suspect that one of the reasons why the use of the VVI-LPM provided unexpectedly preferable clinical outcomes in our present study even though a VVI-LPM is hemodynamically unfavorable compared to a DDD-TPM might be the low complication rate of the patients' VVI-LPMs in the chronic phase. Compared to a DDD-TPM, the implantation of a VVI-LPM could be associated with more lethal complications such as cardiac perforation or vascular injury; however, concerns about lead- or pocket-related complications such as lead dislodgement, lead failure, and infection are expected to disappear or decrease after VVI-LPM implantation, even among very elderly patients. ${ }^{28}$ In fact, the average age of our patients was $82 \pm 7$ years, but no deviceor procedure-related complications were observed during the follow-up periods. The use of a VVI-LPM would thus be reasonable for such a vulnerable cohort, as long as intracardiac echography is applied to help reduce the risk of complications during its implantation.

Our study included 28 (24\%) patients with prior TAVI, of whom 11 patients underwent VVI-LPM implantation due to TAVI-related advanced or complete AVB. The expectation that AVB following TAVI would be transient might lead to an overuse of VVI-LPMs. Although the long-term pacing dependency of AVB following TAVI is inconsistent and varies among previous reports, ${ }^{29-31}$ the $\mathrm{cVP} \%$ at the 12-month or last follow up in our present study was $\geq 80 \%$ in nearly half of the study population. A VVI-LPM should thus not be selected too easily for patients with non-AF bradyarrhythmia following TAVI, unless they have a short life expectancy or a high infection risk.

An AV synchronous LPM will soon be available in Japan, and the need for the desired LPM is likely to greatly increase based on the possibility of diminishing the disadvantages caused by VVI pacing. Nevertheless, as noted above, issues such as TR aggravation and unknown LV dysfunction following VVI-LPM implantation may remain unsettled. After the LPM 'newcomer' device is launched, we should continue to be careful when using an LPM for patients with bradyarrhythmias (especially those with nonAF bradyarrhythmia) because of its potential harmful effect.

\section{Study Limitations}

Our study has several limitations. It was a retrospective observational investigation with loss to follow up, based on a single-center experience. The sample size was thus relatively small, reducing the statistical ability to identify the effects of the device type on clinical outcomes. Second, a PS-matched analysis may pose a risk that we could overlook any clinically important predictor; however, the number of outcomes in our study was low before PS matching as well as after PS matching; that is, 24 events at most; therefore, more patients would be needed to include several potential variables into a multivariable model. Third, the procedures of VVI-LPM implantation, which were performed by several experienced operators, potentially increased the safety of the implantation procedure, resulting in an underestimation of complication rates for the VVI-LPM. Fourth, data on medications such as antiarrhythmic drugs, $\beta$-blockers, and renin-angiotensin-aldosterone inhibitors that could affect clinical prognoses were not available. Finally, the differences in clinical outcomes between the VVI-LPM and DDD-TPM groups observed herein might not have been affected by VVI-LPM implantation itself; rather, the differences may have been affected by the VVI pacing mode. We did not compare the outcomes provided by a VVI-LPM with those afforded by a VVITPM; however, the purpose of this study was to focus on the effect of VVI pacing caused by a VVI-LPM for non-AF bradyarrhythmias; we thus consider our analysis methods appropriate.

\section{Conclusions}

This propensity-matched analysis of elderly Japanese patients with bradyarrhythmia revealed that the implantation of a VVI-LPM for patients with non-AF bradyarrhythmias provided a favorable late $\mathrm{AE}$ rate, but significantly increased the incidence of HF-related rehospitalization compared to that provided by a DDD-TPM over a mid-term follow up.

\section{Disclosures}

None of the authors have any financial disclosures to declare. Y.J.A. is a member of Circulation Journal's Editorial Board.

\section{IRB Information}

St. Marianna University Ethics Committee approved this study (reference no. 5431).

\section{References}

1. Reddy VY, Knops RE, Sperzel J, Miller MA, Petru J, Simon J, et al. Permanent leadless cardiac pacing: Results of the LEADLESS trial. Circulation 2014; 129: 1466-1471.

2. Okuyama K, Izumo M, Sasaki K, Kuwata S, Kaihara T, Watanabe $\mathrm{M}$, et al. Comparison in clinical outcomes between leadless and conventional transvenous pacemaker following transcatheter aortic valve implantation. J Invasive Cardiol 2020; 32: $400-$ 404.

3. El-Chami MF, Clementy N, Garweg C, Omar R, Duray GZ, Gornick CC, et al. Leadless pacemaker implantation in hemodialysis patients: Experience with the Micra Transcatheter Pacemaker. JACC Clin Electrophysiol 2019; 5: 162-170.

4. Lamas GA, Orav EJ, Stambler BS, Ellenbogen KA, Sgarbossa EB, Huang SK, et al. Quality of life and clinical outcomes in elderly patients treated with ventricular pacing as compared with dual-chamber pacing: Pacemaker Selection in the Elderly Investigators. N Engl J Med 1998; 338: 1097-1104.

5. Japanese Circulation Society and Japanese Heart Rhythm Society Joint Working Group. JCS/JHRS 2021 guideline focused update on non-pharmacotherapy of cardiac arrhythmias [in Japanese]. http://www.j-circ.or.jp/guideline/pdf/JCS2021_Kurita_Nogami (accessed September 20, 2021).

6. Nogami A, Kurita T, Abe H, Ando K, Ishikawa T, Imai K, et al. JCS/JHRS 2019 Guideline on non-pharmacotherapy of cardiac arrhythmias. Circ J 2021; 85: 1104-1244.

7. Ritter P, Duray GZ, Zhang S, Narasimhan C, Soejima K, Omar $\mathrm{R}$, et al. The rationale and design of the Micra Transcatheter Pacing Study: Safety and efficacy of a novel miniaturized pacemaker. Europace 2015; 17: 807-813.

8. Tjong FV, Reddy VY. Permanent leadless cardiac pacemaker therapy: A comprehensive review. Circulation 2017; 135: 1458-1470.

9. Zoghbi WA, Adams D, Bonow RO, Enriquez-Sarano, Foster E, Grayburn PA, et al. Recommendations for noninvasive evaluation of native valvular regurgitation: A report from the American Society of Echocardiography developed in collaboration with the Society for Cardiovascular Magnetic Resonance. J Am Soc Echocardiogr 2017; 30: 303-371.

10. Duray GZ, Ritter P, El-Chami M, Narasimhan C, Omar R, Tolosana JM, et al. Long-term performance of a transcatheter pacing system: 12-month results from the Micra Transcatheter 
Pacing Study. Heart Rhythm 2017; 14: 702-709.

11. Soejima K, Asano T, Ishikawa T, Kusano K, Sato T, Okamura $\mathrm{H}$, et al, Micra Transcatheter Pacing Study Group. Performance of leadless pacemaker in Japanese patients vs. rest of the world: Results from a global clinical trial. Circ J 2017; 81: 1589-1595.

12. Cantillon DJ, Dukkipati SR, Ip JH, Exner DV, Niazi IK, Banker RS, et al. Comparative study of acute and mid-term complications with leadless and transvenous cardiac pacemakers. Heart Rhythm 2018; 15: 1023-1030.

13. Piccini JP, El-Chami M, Wherry K, Crossley GH, Kowal RC, Stromberg K, et al. Contemporaneous comparison of outcomes among patients implanted with a leadless vs transvenous singlechamber ventricular pacemaker. JAMA Cardiol 2021; 6: 1187 1195.

14. Ngo L, Nour D, Denman RA, Walters TE, Haqqani HM, Woodman RJ, et al. Safety and efficacy of leadless pacemakers: A systematic review and meta-analysis. J Am Heart Assoc 2021; 10: $\mathrm{e} 019212$.

15. Garg A, Koneru JN, Fagan DH, Stromberg K, Padala SK, ElChami MF, et al. Morbidity and mortality in patients precluded for transvenous pacemaker implantation: Experience with a leadless pacemaker. Heart Rhythm 2020; 17: 2056-2063.

16. Dretzke J, Toff WD, Lip GY, Raftery J, Fry-Smith A, Taylor R. Dual chamber versus single chamber ventricular pacemakers for sick sinus syndrome and atrioventricular block. Cochrane Database Syst Rev 2004; 2004(2): CD003710.

17. Toff WD, Camm AJ, Skehan JD; United Kingdom Pacing and Cardiovascular Events Trial Investigators. Single-chamber versus dual-chamber pacing for high-grade atrioventricular block. $N$ Engl J Med 2005; 353: 145-155.

18. Kruse I, Arnman K, Conradson TB, Rydén L. A comparison of the acute and long-term hemodynamic effects of ventricular inhibited and atrial synchronous ventricular inhibited pacing. Circulation 1982; 65: 846-855.

19. Boon NA, Frew AJ, Johnston JA, Cobbe SM. A comparison of symptoms and intra-arterial ambulatory blood pressure during long term dual chamber atrioventricular synchronous (DDD) and ventricular demand (VVI) pacing. Br Heart J 1987; 58: $34-39$.

20. Appleton CP, Basnight MA, Gonzalez MS. Diastolic mitral regurgitation with atrioventricular conduction abnormalities: Relation of mitral flow velocity to transmitral pressure gradients in conscious dogs. J Am Coll Cardiol 1991; 18: 843-849.

21. Beurskens NEG, Tjong FVY, de Bruin-Bon RHA, Dasselaar KJ, Kuijt WJ, Wilde AAM, et al. Impact of leadless pacemaker therapy on cardiac and atrioventricular valve function through 12 months of follow-up. Circ Arrhythm Electrophysiol 2019; 12: $\mathrm{e} 007124$.

22. Hesselson AB, Parsonnet V, Bernstein AD, Bonavita GJ. Deleterious effects of long-term single-chamber ventricular pacing in patients with sick sinus syndrome: The hidden benefits of dualchamber pacing. J Am Coll Cardiol 1992; 19: 1542-1549.

23. Said S, Cooper CJ, Alkhateeb H, Gosavi S, Dwivedi A, Onate E, et al. Incidence of new onset atrial fibrillation in patients with permanent pacemakers and the relation to the pacing mode. Med Sci Monit 2014; 20: 268-273.

24. Salaun E, Tovmassian L, Simonnet B, Giorgi R, Franceschi F, Koutbi-Franceschi L, et al. Right ventricular and tricuspid valve function in patients chronically implanted with leadless pacemakers. Europace 2018; 20: 823-828.

25. Seo Y, Ishizu T, Ieda M, Ohte N. Right ventricular three-dimensional echocardiography: The current status and future perspectives. J Echocardiogr 2020; 18: 149-159.

26. Zhan Y, Senapati A, Vejpongsa P, Xu J, Shah DJ, Nagueh SF. Comparison of echocardiographic assessment of tricuspid regurgitation against cardiovascular magnetic resonance. JACC Cardiovasc Imaging 2020; 13: $1461-1471$.

27. Morgan DE, Norman R, West RO, Burggraf G. Echocardiographic assessment of tricuspid regurgitation during ventricular demand pacing. Am J Cardiol 1986; 58: 1025-1029.

28. Tachibana M, Banba K, Matsumoto K, Ohara M. The feasibility of leadless pacemaker implantation for superelderly patients. Pacing Clin Electrophysiol 2020; 43: 374-381.

29. Naveh S, Perlman GY, Elitsur Y, Planer D, Gilon D, Leibowitz $\mathrm{D}$, et al. Electrocardiographic predictors of long-term cardiac pacing dependency following transcatheter aortic valve implantation. J Cardiovasc Electrophysiol 2017; 28: 216-223.

30. Meduri CU, Kereiakes DJ, Rajagopal V, Makkar RR, O'Hair $\mathrm{D}$, Linke A, et al. Pacemaker implantation and dependency after transcatheter aortic valve replacement in the REPRISE III trial. J Am Heart Assoc 2019; 8: e012594.

31. Kaplan RM, Yadlapati A, Cantey EP, Passman RS, Gajjar M, Knight BP, et al. Conduction recovery following pacemaker implantation after transcatheter aortic valve replacement. Pacing Clin Electrophysiol 2019; 42: 146-152.

\section{Supplementary Files}

Please find supplementary file(s);

http://dx.doi.org/10.1253/circj.CJ-21-0889 